\title{
Pandemic Novel Coronavirus Outbreak-19: Basic Information and Important Preventive Measures
}

Malik Asif ${ }^{1}$, Shazia Lone ${ }^{2}$, Mushtaq A.Malik ${ }^{1}$, Zaffar Mahdi Dar ${ }^{1}$, Amjad Masood ${ }^{3}$,
Aamir H. Mir ${ }^{4}$, Misbah Ajaz ${ }^{1}$, Saima Shafi ${ }^{1}$, Bisma Rashid ${ }^{1}$ and Asifa Yousuf Wani ${ }^{1}$

${ }^{1}$ Division of Basic Sciences \& Humanities, Faculty of Agriculture-Wadura, India

${ }^{2}$ Department of Environmental Sciences, Govt. Degree College (Boys)-Kupwara, India

${ }^{3}$ Division of Agronomy, Faculty of Agriculture-Wadura-Sopore, India

${ }^{4}$ Division of Soil Sci. \& Agri. Chemistry, Faculty of Agriculture-Wadura, India

${ }^{5}$ Sher-E-Kashmir University of Agricultural Sciences \& Technology of Kashmir, India

*Corresponding author

\section{Keywords}

Coronavirus, Outbreak, Damage, Distribution, Preventive measures

\section{Article Info}

Accepted:

30 March 2020

Available Online:

10 April 2020

\section{A B S T R A C T}

The coronavirus disease (COVID-19) is a highly transmittable and pathogenic viral infection caused by severe acute respiratory syndrome coronavirus 2 (SARS-CoV-2), which emerged in Wuhan, China and spread around the world. The emergence of severe acute respiratory syndrome coronavirus 2 (SARS-CoV-2; previously named 2019 novel coronavirus or 2019-nCoV) disease (COVID-19) in China at the end of 2019 has caused a large global outbreak and is a major public health issue. As of 25 March 2020, data from the World Health Organization (WHO) have shown that more than 21191 and 472000 deaths and confirmed cases respectively have been identified in 46 countries/regions, with $>99 \%$ of cases being detected in China, Italy and Spain. The WHO declared COVID-19 as the $6^{\text {th }}$ public health emergency of international concern. The intermediate source of origin and transfer to humans is not known, however, the rapid human to human transfer has been confirmed widely. There is no clinically approved antiviral drug or vaccine available to be used against COVID-19. However, few broad-spectrum antiviral drugs have been evaluated against COVID-19 in clinical trials, resulted in clinical recovery. SARS-CoV-2 is closely related to two bat-derived severe acute respiratory syndrome-like coronaviruses bat-SL-CoVZC45 and bat-SL-CoVZXC21. It spreads by human -human transmission via droplets and direct contact and infection has been estimated to have mean incubation period of 6.4 days and a basic reproduction number of 2.24-3.58. Among patients with pneumonia caused by SARS-CoV-2, bilateral lung involvement with ground glass opacity was the most common finding from computed topography images of the chest. Public health authorities should keep monitoring the situation very closely. In the current review, we summarize and analyze the epidemiological behaviour and forecasting, symptoms and distribution of the disease outbreak. We also discuss various preventive measures for information of general public across the globe to cope with this viral outbreak. 


\section{Introduction}

Coronavirus is a single-stranded RNA virus with envelope and it has a diameter around $0.1 \mu \mathrm{m}$. belong to the Coronaviridae family in the Nidovirales order. Corona represents crown-like spikes on the outer surface of the virus; thus, it was named as a coronavirus. The virus spreads via droplets from such as cough, direct contact with infected person or contact of hands with contaminated environmental surfaces. These viruses were thought to infect only animals until the world witnessed a severe acute respiratory syndrome (SARS) outbreak caused by SARS-CoV, 2002 in Guangdong, China (1). In 2003, the Chinese population was infected with a virus causing Severe Acute Respiratory Syndrome (SARS) in Guangdong province. The virus was confirmed as a member of the Betacoronavirus subgroup and was named SARSCoV (2, 3). Recently, by the end of 2019, WHO was informed by the Chinese government about several cases of pneumonia with unfamiliar etiology. The outbreak was initiated from the Hunan seafood market in Wuhan city of China and rapidly infected more than 50 peoples. The live animals are frequently sold at the Hunan seafood market such as bats, frogs, snakes, birds, marmots and rabbits(4). On 12 January 2020, the National Health Commission of China released further details about the epidemic, suggested viral pneumonia (4). isolates from the patients, the virus was identified as a novel coronavirus. Moreover, the genetic sequence was also provided for the diagnosis of viral infection. Initially, it was suggested that the patients infected with Wuhan coronavirus induced pneumonia in China may have visited the seafood market where live animals were sold or may have used infected animals or birds as a source of food. However, further investigations revealed that some individuals contracted the infection even with no record of visiting the seafood Frequently clean hands by using alcohol- market. These observations indicated a human to the human spreading capability of this virus, which was subsequently reported in more than 100 countries in the world. The human to the human spreading of the virus occurs due to close contact with an infected person, exposed to coughing, sneezing, respiratory droplets or aerosols. These aerosols can penetrate the human body (lungs) via inhalation through the nose or mouth (5-8). From the sequence-based analysis of So far, the below 6 types of coronaviruses are confirmed to infect humans.

Flu virus among humans: 4 types Human Coronavirus (HCoV) : HCoV-229E, HCoVOC43, HCoV-NL63, HCoV-HKU1

SARS (Severe Acute Respiratory Syndrome) $-\mathrm{CoV}$

MERS (Middle East respiratory syndrome) $\mathrm{CoV}$

Feature of the 2019-nCoV, the novel coronavirus

The 2019-nCoV is a newly identified coronavirus. The common symptom is fever and respiratory symptoms including cough and breathing difficulties. The viral infection also causes pneumonia, severe acute respiratory syndrome and kidney failure for severe cases. The estimated incubation period is ranged from 2 to 11 days but it could be up to 14 days according to the previous other coronavirus experiences. Detailed information is not yet confirmed including the animal source of infection. Thus, it is recommended to stay focus on the reliable latest information such as WHO.

\section{How can we protect ourselves from coronavirus}

WHO recommends below practices to prevent infectious disease transmission including coronavirus.

based hand rub or soap and water. 
When coughing and sneezing cover mouth and nose with flexed elbow or tissuethrow tissue away immediately and wash hands.

Avoid close contact with anyone who has fever and cough.

If you have fever, cough and difficulty breathing seek medical care early and share previous travel history with your health care provider.

When visiting live markets in areas currently experiencing cases of novel coronavirus, avoid direct unprotected contact with live animals and surfaces in contact with animals.

The consumption of raw or undercooked animal products should be avoided. Raw meat, milk or animal organs should be handled with care, to avoid crosscontamination with uncooked foods, as per good food safety practices.

\section{Symptoms of COVID-19}

Symptoms vary from person-to-person with COVID-19. It may produce few or no symptoms. However, it can also lead to severe illness and may be fatal. Common symptoms include: Fever; Breathlessness; Cough

It may take 2-14 days for a person to notice symptoms after infection. (9)

No vaccine is currently available for COVID19. However, scientists have now replicated the virus. This could allow for early detection and treatment in people who have the virus but are not yet showing symptoms. The National Institutes of Health (NIH) suggest that several groups of people have the highest risk of developing complications due to COVID-19. These groups include:

- young children

- people aged 65 years or older

- women who are pregnant
The CDC advise that although there have been reports of complications in young children, these are rare. COVID-19 most commonly produces mild symptoms in children. General symptoms of coronaviruses: Cold- or flu-like symptoms usually set in from 2-4 days after a coronavirus infection and are typically mild. However, symptoms vary from person-to-person, and some forms of the virus can be fatal.

Symptoms may include:

Sneezing; runny nose; fatigue; Cough; fever; sore throat; exacerbated asthma

Scientists cannot easily cultivate human coronaviruses in the laboratory unlike the rhinovirus, which is another cause of the common cold. This makes it difficult to gauge the impact of the coronavirus on national economies and public health. There is no cure for coronaviruses that cause symptoms resembling the common cold. Treatments include self-care and over-the-counter (OTC) medication. People can take several steps, including:

Resting and avoiding overexertion

Drinking enough water

Avoiding smoking and smoky areas

Taking acetaminophen for pain and fever using a clean humidifier or cool mist vaporizer

A doctor can diagnose the virus responsible by taking a sample of respiratory fluids, such as mucus from the nose, or blood.

\section{Types}

Coronaviruses belong to the subfamily Coronavirinae in the family Coronaviridae.

Different types of human coronaviruses vary in how severe the resulting disease becomes, 
and how far they can spread. Doctors currently recognize seven types of coronavirus that can infect humans.

\section{Common types include}

229E (alpha coronavirus); NL63 (alpha coronavirus); OC43 (beta coronavirus); HKU1 (beta coronavirus).

Rarer strains that cause more severe complications include MERS-CoV, which causes Middle East respiratory syndrome (MERS), and SARS-CoV, the virus responsible for severe acute respiratory syndrome (SARS). In 2019, a new strain called SARS-CoV-2 started circulating, causing the disease COVID-19.

\section{Transmission}

Limited research is available on how $\mathrm{HCoV}$ spreads from one person to the next. However, researchers believe that the viruses transmit via fluids in the respiratory system, such as mucus. Coronaviruses can spread in the following ways:

Coughing and sneezing without covering the mouth can disperse droplets into the air.

Touching or shaking hands with a person who has the virus can pass the virus between individuals.

Making contact with a surface or object that has the virus and then touching the nose, eyes, or mouth.

Some animal coronaviruses, such as feline coronavirus (FCoV), may spread through contact with feces. However, it is unclear whether this also applies to human coronaviruses.

Coronaviruses will infect most people at some time during their lifetime.

Coronaviruses can mutate effectively, which makes them so contagious.
To prevent transmission, people should stay at home and rest while symptoms are active. They should also avoid close contact with other people.Covering the mouth and nose with a tissue or handkerchief while coughing or sneezing can also help prevent transmission. It is important to dispose of any tissues after use and maintain hygiene around the home.

\section{SARS}

SARS is a contagious disease that develops after infection by the SARS-CoV coronavirus. Typically, it leads to a life threatening form of pneumonia. During November 2002, the virus started in the Guangdong Province in southern China, eventually reaching Hong Kong. From there, it rapidly spread around the world, causing infections in more than 24 countries.

SARS-CoV can infect both the upper and lower respiratory tracts. The symptoms of SARS develop over the course of a week and start with a fever. Early on in the condition, people develop flu-like symptoms, such as:

Dry coughing; Chills; diarrhea; Breathlessness; Aches

Pneumonia, a severe lung infection, usually develops. At its most advanced stage, SARS causes failure of the lungs, heart, or liver. According to the CDC, authorities marked 8,098 people as having contracted SARS during its outbreak. Of these, 774 infections were fatal. This equates to a mortality rate of 9.6\%. Complications are more likely in older adults, and half of all people over 65 years of age who became ill did not survive. Authorities eventually controlled SARS in July 2003. However, it can still occur after infection with SARS-CoV. 


\section{Situation assessment}

Epidemiological overview as of $25^{\text {th }}$ of March, 2020

A total of 472000 confirmed cases of 2019-nCoV have been reported worldwide (WHO-2020)

Of the total cases reported, maximum cases have been reported from China, Italy and Spain

In China, $60.5 \%$ of all cases since the start of the outbreak have been reported from Hubei Province. The remaining $39.5 \%$ of cases have been reported from 33 provinces, regions, and cities. After Hubei Province, the second largest number of cases has been reported from Zhejiang Province (599 cases)

21191 deaths across the globe have been reported to date. Epidemiological evidence shows that 2019-nCoV can be transmitted from one individual to another. During previous outbreaks due to other coronaviruses, including Middle-East respiratory syndrome coronavirus (MERS$\mathrm{CoV}$ ) and the Severe Acute Respiratory Syndrome coronavirus (SARS-CoV), humanto-human transmission most commonly occurred through droplets, personal contact, and contaminated objects (fomites). The modes of transmission of 2019-nCoV are likely to be similar. The precise zoonotic (animal) origin of the $2019-\mathrm{nCoV}$ is still uncertain. The virus has been identified in environmental samples from a live animal market in Wuhan, and some human cases have been epidemiologically linked to this market. Other coronavirus, such as SARS and MERS, are also zoonotic, and can be transmitted from animals (civet cats and dromedary camels, respectively) to humans.

\section{Risk assessment}

WHO assesses the risk to be very high for
China, Italy, Spain and European countries, high at the regional level, and high at the global level.

\section{Factors taken into consideration include}

\section{Likelihood of further spread}

Human-to-human transmission, including transmission within families and healthcare settings, has been confirmed within Wuhan, and in several cities outside China. The outbreak continues to grow within China at a rapid rate, and now affects all 31 provinciallevel administrative regions.

Ordinarily high volumes of domestic and international travel have been increased further by travel linked to Lunar New Year celebrations. Imported cases continue to be reported internationally, with several reported cases of secondary transmission now confirmed in countries outside of China. Limited testing capacity in many countries globally, non-specific symptoms of 2019-nCoV acute respiratory disease (the disease caused by 2019-nCoV infection), and co-circulation of other respiratory pathogens are factors that can complicate efforts to detect the virus quickly.

\section{Potential impact on human health}

The virus can cause severe illness and death, although most cases appear to be mild. However, many uncertainties remain, including the full extent of the current outbreak within China, and the full clinical spectrum of illness, including the prevalence of mildly symptomatic cases.

\section{Effectiveness of current preparedness and response measures}

China has dedicated substantial resources to public health control measures and clinical management, and has taken action that has 
included the quarantine of cities, and the widespread suspension of transport links between population centres. It will be important to continually assess the extent to which measures are effective and the need to adapt measures as the situation evolves. Up to now, countries that have reported an imported case have demonstrated efficient and effective disease surveillance and response measures. However, some countries are less prepared to detect and respond to an imported case. Rumours, misconceptions, and misinformation disseminated online via social media can have a negative impact on response measures and health-seeking behaviors.

\section{Recommendations of the emergency committee on 30 January 2020}

Director-General of WHO declared the 2019-nCoV outbreak a public health emergency of international concern under the International Health Regulations (IHR) (2005), following advice from the Emergency Committee. The Director-General and Emergency Committee issued temporary recommendations1 to the People's Republic of China and to other countries. The Emergency Committee also provided advice to $\mathrm{WHO}$, and welcomed a forthcoming WHOled multidisciplinary and multi-partner technical mission to China. The mission will review and support efforts to investigate the animal source of the outbreak, the clinical spectrum of the disease and its severity, the extent of human-to-human transmission in the community and in healthcare facilities, and efforts to control the outbreak. This mission will provide information to the international community to aid in understanding the situation, its impact, and effective public health measures to respond to the virus. The Committee recommended that WHO should continue to use its networks of technical experts to assess how best this outbreak can be contained globally, and intensify support for preparation and response, especially in vulnerable countries and regions.

\section{Response strategy}

The overall goal of the strategic preparedness and response plan is to stop further transmission of 2019-nCoV within China and to other countries, and to mitigate the impact of the outbreak in all countries. Taking the above into account, the strategic objectives of the plan are to:

Limit human-to-human transmission, including reducing secondary infections among close contacts and healthcare workers, preventing transmission amplification events, and preventing further international spread from China;

Identify, isolate, and care for patients early, including providing optimized care for infected patients;

Identify and reduce transmission from the animal source;

Address crucial unknowns regarding clinical severity, extent of transmission and infection, treatment options, and accelerate the development of diagnostics, therapeutics, and vaccines;

Communicate critical risk and event information to all communities, and counter misinformation;

Minimize social and economic impact through multisectoral partnerships.

\section{These objectives can be achieved by:}

Rapidly establishing international coordination to deliver strategic, technical, and operational support through existing mechanisms and partnerships.

Scaling up country preparedness and response 
operations, including strengthening readiness to rapidly identify, diagnose and treat cases; identification and follow-up of contacts when feasible (with priority given to high-risk settings such as healthcare facilities); infection prevention and control in healthcare settings; implementation of health measures for travelers; and awareness raising in the population though risk communication and community engagement.

Accelerating priority research and innovation to support a clear and transparent global process to set research and innovation priorities to fast track and scale-up research, development, and the equitable availability of candidate therapeutics, vaccines, and diagnostics. This will build a common platform for standardized processes, protocols and tools, to facilitate multidisciplinary and collaborative research integrated with the response. The response strategy is based on several planning assumptions. Owing to the considerable uncertainty surrounding the extent of the outbreak within China, the transmissibility of the virus, and the clinical spectrum of the disease, it will be necessary to regularly update these assumptions as gaps in our knowledge of the disease are filled. The current response plan assumes that human-tohuman transmission takes place, and that it may be amplified in specific settings, including healthcare facilities.

Also assume that human-to-human transmission is widespread within Hubei, and possibly other population centres in China. It is expected that cases will continue to be exported to other countries while the outbreak continues in China. While the response emphasis will be to rapidly identify and isolate imported cases, there is a risk of clusters of cases caused by localized community transmission outside China. In some cases, countries may require operational assistance to strengthen their capacity to detect and respond to these imported cases.
However, there remain significant uncertainties around the potential for more widespread transmission outside China, and it will therefore be necessary to have contingency plans in place to mitigate the challenges this would present.

\section{Risk communication and managing the infodemic}

The 2019-nCoV outbreak and response has been accompanied by an infodemic an overabundance of information - some accurate and some not - that makes it hard for people to find trustworthy sources and reliable guidance when they need it. Due to the high demand for timely and trustworthy information about 2019-nCoV, WHO technical risk communication and social media teams have been working closely to track and respond to myths and rumours. WHO and partners are working 24 hours a day to identify the most prevalent rumours that can potentially harm the public's health, such as false prevention measures or cures. These myths are then refuted with evidence-based information.

WHO and partners are making public health information and advice on the 2019-nCoV, including myth busters, available on social media channels (including Weibo, Twitter, Facebook, Instagram, LinkedIn, Pinterest) and organizational websites. In addition, an expanding group of global response organizations such as the United Nations Children's Fund (UNICEF) and the International Federation of Red Cross and Red Crescent Societies (IFRC) are coordinating efforts with WHO to ensure that biomedical recommendations can be applied at the community level. These organizations are active at the global, regional, and country level to ensure that affected populations have a voice and are part of the response. Ensuring that global recommendations and 
communication are tested, adapted, and localized will help countries better control the 2019-nCoV outbreak.

\section{Laboratory and diagnostics}

Partners will work together to strengthen global diagnostic capacity for 2019-nCoV detection in order to improve surveillance and track the spread of disease. Public health efforts to limit spread and strengthen disease control in countries with imported cases depend critically on the ability to detect the pathogen. WHO and partners have activated a network of specialized referral laboratories with demonstrated expertise in the molecular detection of coronaviruses. These international laboratories can support national laboratories to confirm new cases. WHO and partners will work to ensure the availability of tests, including through the screening validation of current academic and commercially available assays. WHO will work with commercial and non-commercial agencies with the capacity to manufacture and distribute newly-developed 2019-nCoV molecular assays. WHO will begin supporting its regional and country offices to make these tests available from the first week of February 2020 .

\section{Technical expertise and guidance}

On 10 January, WHO published a range of information for all countries on how they can prepare for and respond cases and clusters of 2019-nCoV, including handling possible cases, how to identify and monitor sick people, collect and test samples, treat patients, prevent onward spread, control transmission in healthcare facilities, maintain adequate stocks of supplies, and communicate with the public about 2019-nCoV infection. Guidance was developed from existing materials for MERS-CoV and updated with input from a global network of partners from affected countries, and global partners with expertise in laboratory, clinical management, infection prevention and control, mathematical modelling, risk communication, and community engagement. The full WHO technical guidance pack including the following guidance materials is reviewed and updated regularly as new information becomes available:

Surveillance case definitions for human infection with 2019-nCoV;

Interim guidance on laboratory testing of human cases suspected of 2019-nCoV infection, and protocols for molecular testing for 2019-nCoV;

Clinical management of severe acute respiratory infection when 2019-nCoV infection is suspected

Infection prevention and control during healthcare when 2019-nCoV infection is suspected; - Home care for patients with suspected 2019-nCoV infection presenting with mild symptoms and management of contacts;

Guidance on risk communication and community engagement and initial response;

Recommendations on the prevention of transmission from animals to humans;

Early investigations of suspected cases. A country readiness checklist was also made available to support teams to review their capacities to detect, manage, and respond to suspect cases and clusters. A disease commodity package for 2019-nCoV outlines the supplies needed for surveillance, laboratory analysis, clinical management, and infection prevention and control. Global coordination of technical expertise for laboratory, clinical management, infection 
prevention and control, risk communication and community engagement, and mathematical modelling will continue to gather real-time information and be incorporated into available guidance.

\section{Pandemic supply chain coordination}

Partners will use their expertise in quantifying global needs according to various outbreak scenarios, and in the coordination of procurement and supply mechanisms, to ensure that countries and populations most in need are assured of access to crucial supplies and commodities.

In practice, this will entail the gathering and analysis of all available information from partner networks to establish supply scenarios based on a prioritized list of countries and population affected, number of patients to be treated, and population to be protected. This analysis will inform the deployment necessary health logistics personnel to regional and country level locations to support logistical efforts. The Pandemic Supply Chain Network and coordination mechanism will be activated to ascertain the current and forecast availability of crucial supplies in order to coordinate the distribution of appropriate supplies to where they are most needed.

Where necessary, partners will establish appropriate operational mechanisms to secure critical supplies with private sector manufacturers and logistics providers to provide country-level support, and negotiate commitments from manufacturers and wholesalers to ensure procurement of critical supplies, prevent stockouts, and mitigate market disruptions and hoarding. Contingency plans will be developed to mitigate disruption to supplies of food and essential commodities (non-medical) supplies in the event that vulnerable countries are affected by widespread transmission.

\section{Scaling up country readiness and response operations}

Beyond establishing international coordination and operational support, it is crucial to scale up country preparedness and response operations, including the rapid identification, diagnosis and management of cases, identification and follow up of contacts when feasible (with priority given to high-risk settings such as healthcare facilities), infection prevention and control in healthcare settings, implementation of health measures for travelers, and awareness raising in the population though risk communication and community engagement. All countries are at risk and need to prepare for 2019-nCoV. Partners will prioritize countries with weak health systems and significant gaps in preparedness capacity for technical and operational support. A rapid risk and vulnerability mapping has been done based on country capacity as measured through Member States annual reporting of IHR (2005) core capacities, and the likelihood of importation of cases based in international travel volumes from high-risk cities China in January 2020.

\section{Country-level coordination}

In national risk management, the government is the natural leader for overall coordination and communication efforts. Building on global risk assessments to inform national risk assessments and to decide on actions needed, the national government should help public and private agencies and organizations by providing guidance, planning assumptions and making appropriate modifications to the laws or regulations at all levels and sectors to enable an effective response. These efforts are supported by WHO and other UN organizations under IHR (2005), and through the Cluster-coordination approach where relevant. The process should build on existing 
public health emergency contingency, preparedness and response plans, including for pandemic influenza. A key step is the activation of existing national emergency response committee(s) to take the lead in coordination of these functions, and to provide the forum for partners to be involved in response operations.

A Public Health Emergency of International Concern will test the resilience of nations, businesses, and communities, depending on their capacity to respond. No single agency or organization can prepare for or respond to such an event on its own. Inadequate or uncoordinated preparedness of interdependent public and private organizations will reduce the ability of the health sector to control disease spread. A comprehensive approach to risk management is therefore required and must take a whole-of-society and whole of government approach. All entities need to consider supportive financial resources for response operations, and to develop plans for essential service continuity and recovery operations.

\section{Risk communication and community engagement}

Country risk communication and community engagement is a critical public health intervention in all countries. Countries should prepare to communicate rapidly, regularly and transparently with the population. All countries should prepare existing public health communication networks, media and community engagement staff to be ready for a possible case, and for the appropriate response if this happens. Countries should coordinate communications with other response organizations and include the community in response operations. Partners stand ready to coordinate with partners to support countries in their communication and community engagement response.

\section{Surveillance}

National authorities must notify WHO of detected cases under IHR (2005), and undertake case-based and/or aggregated reporting as per global surveillance guidance. Partners will provide any support necessary to enable authorities to fulfil these obligations. Disaggregated data on age, sex, pregnancy status and outcome (as appropriate) should be reported. It will be important to enhance existing respiratorydisease-surveillance systems, including indicator-based surveillance, community event-based surveillance, and sentinel surveillance (e.g., Severe Acute Respiratory Infection and Influenza-like illness), and establish active case finding at points of entry, health facilities and in communities. It may be necessary to engage the private sector in case identification and surveillance where appropriate.

\section{Points of entry}

Countries should establish or update a multisectoral contingency plan at points of entry, integrated with other emergency operational plans at point of entry, covering relevant stakeholders, and conduct simulation exercises to assess the operational applicability of the plan. In terms of physical infrastructure it will be necessary to designate an appropriate place, separate from other travelers, where travelers who meet the definition of a suspected 2019-nCoV case can be interviewed in a safe (including provision of personal protective equipment) and dignified way away from other travelers.

Any suspected cases should be granted access to medical follow up by establishing arrangements with local medical facilities for isolation, treatment, and other support services that may be required, including for sample collection and transport for laboratory testing. Protocols and referral pathways 
should be established to guide safe transport of patients and their close contacts to designated medical facilities. Points of entry authorities should also establish mechanisms and procedures for communicating information (see also risk communication above) to travellers via travel health clinics, travel agencies, tour operators and at points of entry, about the disease, preventative measures to reduce the general risk of acute respiratory infections, and how and where to seek medical attention.

\section{Rapid response teams}

National rapid-response teams should be trained and equipped for investigation of suspected 2019-nCoV cases and initial treatment where appropriate. This will require the preparation and dissemination of case investigation protocols (as per WHO guidance) and supplies, establishment of a system for contact tracing and monitoring, and the implementation of a communitybased surveillance mechanism.

\section{Infection prevention and control}

Infection prevention and control (IPC) measures are absolutely essential to ensure healthcare workers are protected from infection with 2019-nCoV and amplification events in healthcare facilities. An IPC programme at national and facility level with a dedicated and trained team, or at least an IPC focal point, should be in place and supported by the national authorities and facility senior management.

In countries where IPC is sub-optimal or limited, partners will need to support national authorities to ensure that at least minimum requirements for IPC are in place as soon as possible, both at the national and facility level, and to gradually progress to achievement of all requirements of the IPC core components according to local priority plans. Partners should support national authorities to undertake a risk assessment of IPC capacity at all levels of the healthcare system (includes availability of triage and appropriately ventilated isolation rooms) and, on the basis of this assessment, define a referral pathway in collaboration with case management capacity. Particular attention should be given to ensuring IPC compliance with basic IPC principles at the first point of care (usually primary care).

Capacity for triage, early recognition, standard precautions, isolation capacity, and referral procedures should align with WHO IPC guidance on 2019-nCoV. If supplies are needed to implement recommended protocols (e.g., hand hygiene resources, personal protective equipment, environmental cleaning, and waste management), partners should assist national authorities in procurement and supply where appropriate.

Partners will support national efforts to identify IPC surge capacity (numbers and competence) that can be deployed to strategic locations. It will be important to monitor, analyse, and feedback to relevant stakeholders any data on healthcare-associated infections in patients and in healthcare workers to ensure such infections are prevented.

\section{Logistics, procurement, and supply management}

Logistics, procurement, and supply chain management must be integrated across national preparedness and response efforts. This includes ensuring that logistics and supply management addresses needs and risks arising from epidemiological events, communication needs, and geopolitical events, all of which may have a substantial impact on the market's ability to manufacture necessary supplies, and to distribute them 
equitably and efficiently to the appropriate locations.

Accelerating priority research and innovation

The 2019-nCoV outbreak presents many urgent questions. How easily is the virus transmitted? What is the zoonotic source? How severely does the virus affect patients, and who is most at risk? And what countermeasures - diagnostics, vaccines, and therapeutics - might be developed to stop the virus spreading, improve treatment outcomes, and strengthen our ability to rapidly detect the disease? It is crucial that the international community agrees on priority areas for research and innovation, and the most efficient ways to address them. WHO will convene all stakeholders to address these challenges in the following three key areas.

\section{Enhancing global coordination of all} relevant stakeholders

The world needs an emergency mechanism to coordinate all the different stakeholders in global research and development (R\&D), from academics and industry to national governments, civil society groups, and nongovernmental organizations. That mechanism already exists: the R\&D Blueprint Global Coordination Mechanism (GCM) is a voluntary framework to facilitate information sharing and incentivize funders, product developers, and researchers to share evidence about candidate vaccines, therapeutics, and diagnostics in the pipeline that could be brought to bear against 2019-nCoV.

Such coordination is essential to make sure that any gaps in the research agenda are filled, and any duplication of effort is avoided. The GCM has been activated
Scaling up country preparedness and response operations

Resource requirements for scaling up country preparedness and response operations are based on:

an assessment of the capacities required by countries to prepare and respond to 2019$\mathrm{nCoV}$ for a given risk level; and

the current country preparedness capacity. The capacities required by countries to prepare and respond to 2019-nCoV are based on if a country has:

Clusters of community transmission of 2019$\mathrm{nCoV}$

Clusters of local transmission of 2019-nCoV

Imported cases of 2019-nCoV

High-risk of imported cases 4 of 2019-nCoV.

The capacities required by countries to prepare and respond to 2019-nCoV will be updated on a regular basis based on the evolving epidemiological situation and risk of spread.

The current country preparedness capacity is based on IHR (2005) State Parties SelfAssessment Annual Reporting Tool that measures reported core capacities in respect of health security including surveillance, laboratory, and emergency coordination capacity and ranks capacity on a 1-5 scale. Country preparedness capacity to respond to 2019-nCoV will be updated based on specific country-level gap analyses. 
Conclusion and perspective are as follows:

The novel coronavirus originated from the Hunan seafood market at Wuhan, China where bats, snakes, raccoon dogs, palm civets, and other animals are sold, and rapidly spread up to 109 countries. Until now, no promising clinical treatments or prevention strategies have been developed against human coronaviruses. However, the researchers are working to develop efficient therapeutic strategies to cope with the novel coronaviruses. Various broad-spectrum antivirals previously used against influenza, SARS and MERS coronaviruses have been evaluated either alone or in combinations to treat COVID-19 patients, mice models, and clinical isolates. While the decision of bring back the nationals from infected area by various countries and poor screening of passengers become the leading cause of spreading virus in others countries. Most importantly human coronaviruses targeting vaccines and antiviral drugs should be designed that could be used against the current as well as future epidemics. Moreover, the public need to be educated about health and hygiene through electronic media under such a grim situation. At the juncture social distancing and self-quarantine especially in the developing countries seems to be the only alternative to get rid off from this viral outbreak.

\section{References}

1.Zhong N, Zheng B, Li Y, Poon L, Xie Z, Chan $\mathrm{K}$, et al., Epidemiology and cause of severe acute respiratory syndrome (SARS) in Guangdong,
People's Republic of China, in February, 2003. The Lancet. 2003; 362(9393): 1353-8.

2.Peiris J, Guan Y, Yuen K. Severe acute respiratory syndrome. Nature medicine. 2004; 10(12): S88-S97.

3.Pyrc K, Berkhout B, Van Der Hoek L. Identification of new human coronaviruses. Expert review of antiinfective therapy. 2007;5(2):245-53.

4.Wang C, Horby PW, Hayden FG, Gao GF. A novel coronavirus outbreak of global health concern. The Lancet. 2020.

5.Phan LT, Nguyen TV, Luong QC, Nguyen TV, Nguyen HT, Le HQ, et al., Importation and human-to-human transmission of a novel coronavirus in Vietnam. New England Journal of Medicine. 2020.

6.Riou J, Althaus CL. Pattern of early humanto-human transmission of Wuhan 2019 novel coronavirus (2019nCoV), December 2019 to January 2020. Eurosurveillance. 2020;25(4).

7.Parry J. China coronavirus: cases surge as official admits human to human transmission. British Medical Journal Publishing Group; 2020.

8.Li Q, Guan X, Wu P, Wang X, Zhou L, Tong $\mathrm{Y}$, et al., Early transmission dynamics in Wuhan, China, of novel coronavirus-infected pneumonia. New England Journal of Medicine. 2020.

9.2019 Novel Coronavirus (2019-nCoV): Strategic Preparedness and Response Plan-WHO-Switzerland. 


\section{How to cite this article:}

Malik Asif, Shazia Lone, Mushtaq A.Malik, Zaffar Mahdi Dar, Amjad Masood, Aamir H. Mir, Misbah Ajaz, Saima Shafi, Bisma Rashid and Asifa Yousuf Wani. 2020. Pandemic Novel Coronavirus Outbreak-19: Basic Information and Important Preventive Measures. Int.J.Curr.Microbiol.App.Sci. 9(04): 3187-3200. doi: https://doi.org/10.20546/ijcmas.2020.904.371 